\title{
A Novel Robust Sliding Mode Controller for Half-bridge Converter
}

\author{
Lijun Hang*, Sensen Liu*, Zhengyu Lu*, and Miguel Castilla** \\ *:College of Electrical Engineering, Zhejiang University, CHINA, 310027 \\ **: Universitat Politècnica de Catalunya, SPAIN
}

\begin{abstract}
This paper proposes one novel sliding mode controller for the Half-bridge converter to reduce the line and load disturbance. Based on the bilinear model of the Half-bridge DC/DC converter, the small signal model and the design consideration of the sliding mode controller for this converter are deduced and presented. The controller is simply implemented in the analog controller board. The validation of the controller is verified both by theory analysis and experimental results. By applying the novel sliding mode controller, the Half-bridge converter can achieve good transient response.
\end{abstract}

Index Terms-Sliding mode, Half-bridge, DC/DC, Power Electronics

\section{INTRODUCTION}

The sliding mode (SM) control approach is recognized as an efficient tool to design robust controllers for the complex high-order nonlinear dynamic plant operating under uncertain conditions. The major advantage of sliding mode is low sensitivity to the plant parameter variations and disturbances which eliminates the necessity of exact modeling. So the SM controller is a type of nonlinear controller well known for its robustness, stability, and good regulation properties in large-signal operations [1],[2]. It is deemed to be a better candidate than other nonlinear controllers for its relative ease of implementation. As the only admissible operation mode, it is implied that the control actions of the sliding mode control are discontinuous state functions. This kind of control mode can easily be implemented by the conventional power converters with "on-off" control action. In particular, the fixed-frequency pulse width modulation (PWM) based SM controllers are found to be more suited for practical implementation in power converters [2]. Recent successes of its application in DCDC converters have been reported in [3]-[4]. It is also illustrated in these papers that better regulation of the converters over a wide range of operating condition is achieved with the SM controllers as compared to conventional PWM controllers.

On another side, DC/DC converters are widely used in regulated switch-mode DC power supplies and DC motor drive applications. Often the input to these converters is an unregulated DC voltage, which may have been obtained by rectifying the line voltage, and therefore will fluctuate due to changes in the line-voltage magnitude. Numerous analog and digital control methods for dc-dc

This work was supported by National Natural Science Foundation of CHINA(50907059). converters have been proposed and some have been adopted by industry including voltage-mode and currentmode control techniques. It is of great interest to compare the dynamic response of these control methods as well as their advantages and disadvantages. Nowadays, the Halfbridge DC/DC topology is a preferred candidate for the low voltage application, both improved new technique and control method are developed for the converter[5]-[7].

This paper presents a novel sliding mode controller for the Half-bridge converter. The controller is robust and flexible for the converter. First, the small signal model and the controller design will be presented. Then, the validity of the controller will be verified by simulating and experimental results.

\section{Small Signal Model of The Controller AND DESIGN CONSIDERATION}

Fig. 1 shows the typical half-bridge topology. The characteristic between the output and the duty ratio is illustrated in Fig.2. In order to achieve the specified voltage, there are two operating regions. The converter can be described by the following bilinear model (1)(4) [8].

$$
\begin{aligned}
& L_{m} \frac{d i_{m}}{d t}=E \cdot u-v_{c 2} \\
& C_{e q} \frac{d v_{C 2}}{d t}=C_{1} \cdot \frac{d E}{d t}+i_{m}+n \cdot(2 u-1) \cdot i_{L} \\
& L_{o} \frac{d i_{L}}{d t}=n \cdot\left[E \cdot u-(2 u-1) v_{C 2}\right]-v_{o} \\
& C_{o} \frac{d v_{o}}{d t}=i_{L}-\frac{v_{o}}{R_{L}}
\end{aligned}
$$

Wherein $C_{e q}=C_{1}+C_{2}, u$ is the control variable. As explained in Fig. 1, when $u=1, \mathrm{~S}_{1}$ and $\mathrm{S}_{3}$ are turned on; when $u=0, \mathrm{~S}_{2}$ and $\mathrm{S} 4$ are turned on. As shown in equation (1) to (4), in the converter of HB there are four state variables. In order to apply the sliding mode controller, the following full surface of a $\mathrm{HB}$ converter can be obtained:

$$
\begin{aligned}
& S=\left(V_{C 2 S S}-v_{c 2}\right) \cdot K_{1}+\left(I_{m S S}-i_{m}\right) \cdot K_{2} \\
& +\left(I_{L S S}-i_{L}\right) \cdot K_{3}+\left(V_{r e f}-v_{o}\right) \cdot K_{4}
\end{aligned}
$$

In general, $i_{m}$ is not easy to sense, so normally, this state variable is eliminated. Wherein

$$
i_{L}=C_{o} \frac{d v_{o}}{d t}+\frac{v_{o}}{R}
$$


In order to avoid the steady state error in $v_{o}$, normally the output voltage error is controlled with integrator, i.e. $K_{i} \int\left(V_{r e f}-v_{o}\right) d t$. So we propose a full normal surface for HB converter:

$$
\begin{aligned}
& S=k_{p} \cdot\left(V_{r e f}-v_{o}\right)+k_{i} \int_{-\infty}^{t}\left(V_{r e f}-v_{o}\right)+ \\
& k_{d} \frac{d\left(V_{r e f}-v_{o}\right)}{d t}+\left(V_{C 2 S S}-v_{C 2}\right) \cdot K_{4}
\end{aligned}
$$

The sliding surface is easy to implement. Only two voltages should be sensed, the controller can be implemented in analog mode.

Generally, in SM controller, the reaching condition $S \cdot \dot{S}<0$ should be followed, the control criterion is obtained as

$$
\begin{aligned}
& u=u^{+}, S>0 \rightarrow \frac{d S}{d t}<0, \\
& u=u^{-}, S<0 \rightarrow \frac{d S}{d t}>0
\end{aligned}
$$

In $\mathrm{SM}$ controller, the converter dynamics is forced to evolve around the sliding surface $S$ according to the invariance condition $\dot{S}=0$, so the following equation can be concluded :

$$
\begin{aligned}
& \dot{S}=\frac{d S}{d t}=k_{p} \frac{d v_{o}}{d t}+k_{i} \cdot\left(v_{o}-V_{r e f}\right) \\
& +k_{d} \frac{d^{2} v_{o}}{d t}-k_{4} \frac{d v_{c 2}}{d t}=0
\end{aligned}
$$

From where, the dynamic of $v_{c 2}$ also can be obtained as follows:

$k_{4} \frac{d v_{c 2}}{d t}=k_{p} \frac{d v_{o}}{d t}+k_{i} \cdot\left(v_{o}-V_{r e f}\right)+k_{d} \frac{d^{2} v_{o}}{d t}$

By applying the novel sliding mode controller (7), the system has novel equivalent external dynamic (10). As shown in (1), (2), (3) and (4), the whole system can be divided as two subsystems: external dynamics which are given by (4) and (10), and the internal dynamics which are given by (1) and (3). It is obvious that the internal

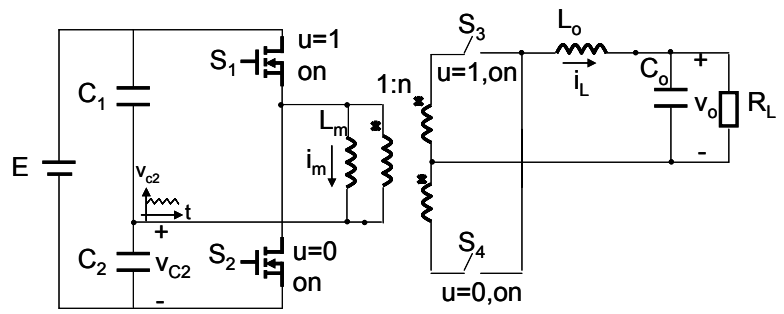

Figure 1: Main circuit of Half-bridge Converter

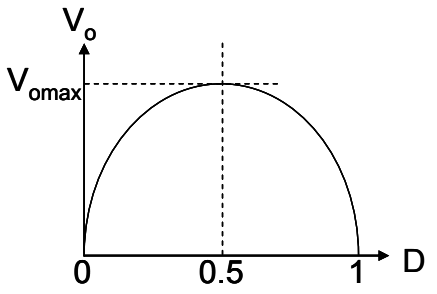

Figure 2: Relation of $\mathrm{V}_{\text {omax }}$ and duty-ratio (D)

dynamics and the external dynamics are coupled. Therefore, the stability analysis of the whole system can be performed by studying the two subsystems together. In the following part, the study of the whole system will be performed.

\section{1)Derivation of the small signal model}

It is supposed that the system is in the stable state. From (2) and (10), the equivalent control action can be obtained as follows:

$$
u_{e q}=\frac{M_{C}-i_{m}}{2 \cdot u \cdot i_{L}}+\frac{1}{2}
$$

Where,

$$
M_{c}=\frac{C_{e q}}{k_{4}} \cdot\left[k_{p} \frac{d v_{o}}{d t}+k_{i} \cdot\left(v_{o}-V_{r e f}\right)+k_{d} \frac{d^{2} v_{o}}{d t}\right] \text {. }
$$

It is supposed that the system is in the stable state. Substitute (11) into (2), make perturbation for (1)-(4), and finally, the small signal state variable equations can be obtained.

In addition, in the stable state, the following equations can be obtained from (1) to (4)

$$
\begin{gathered}
I_{m}{ }^{*}=n \cdot I_{L}{ }^{*} \cdot\left(1-2 \cdot u_{e q}{ }^{*}\right) \\
E \cdot u_{e q}{ }^{*}=V_{c 2}{ }^{*} \\
n \cdot E \cdot u_{e q}{ }^{*}=n \cdot V_{c 2}{ }^{*} \cdot\left(1-2 \cdot u_{e q}{ }^{*}\right)+V_{r e f} \\
I_{L}{ }^{*}=\frac{V_{r e f}}{R}
\end{gathered}
$$

Wherein, $I_{m}{ }^{*}, I_{L}{ }^{*}, u_{e q}{ }^{*}$ and $V_{c 2}{ }^{*}$ are the stable value. By using (1'), (2'), (3') and (4'), the small signal model can be simplified. In the small signal region, finally the small signal model can be written as follows:

$$
\begin{gathered}
\frac{d \widehat{v_{o}}}{d t}=\frac{1}{C}\left(\widehat{\hat{i_{L}}}-\frac{\widehat{v_{o}}}{R}\right) \\
\frac{d \hat{i_{L}}}{d t}=K \cdot K_{o} \cdot \widehat{v_{o}}+K \cdot K_{L} \cdot \widehat{i_{L}}+K \cdot k_{E} \cdot \widehat{i_{m}}+2 \cdot n \cdot I_{m}^{*} \cdot \widehat{v_{c 2}} \\
\frac{d \hat{i_{m}}}{d t}=\frac{1}{2 \cdot n \cdot L_{m} \cdot I_{L}^{*}}\left(F_{o} \cdot \widehat{v_{o}}+F_{L} \cdot \widehat{i_{L}}+F_{m} \cdot \widehat{i_{m}}+F_{c 2} \cdot \widehat{v_{c 2}}\right)
\end{gathered}
$$

Wherein,

$$
\begin{aligned}
& k_{E}=2 \cdot n \cdot V_{c 2}{ }^{*}-n \cdot E \\
& K=\frac{1}{2 \cdot n \cdot L \cdot I_{L}{ }^{*}+C_{e q} \cdot k_{d} \cdot k_{E} /\left(C \cdot k_{4}\right)},
\end{aligned}
$$




$$
\begin{aligned}
& K_{o}=\frac{C_{e q} \cdot k_{p} \cdot k_{E}}{R \cdot C \cdot k_{4}}-\frac{C_{e q} \cdot k_{i} \cdot k_{E}}{k_{4}}-\frac{C_{e q} \cdot k_{d} \cdot k_{E}}{R^{2} \cdot C^{2} \cdot k_{4}}-2 \cdot n \cdot I_{L}^{*}, \\
& K_{I}=\frac{C_{e q} \cdot k_{d} \cdot k_{E}}{R \cdot C^{2} \cdot k_{4}}-\frac{C_{e q} \cdot k_{p} \cdot k_{E}}{C \cdot k_{4}}+n^{2} \cdot E-2 \cdot n \cdot V_{o}^{*}, \\
& F_{o}=\frac{E \cdot C_{e q} \cdot k_{d}}{C \cdot k_{4}} \cdot K \cdot K_{o}+\frac{E \cdot C_{e q} \cdot k_{d}}{R^{2} \cdot C^{2} \cdot k_{4}}, \\
& +\frac{E \cdot C_{e q} \cdot k_{i}}{k_{4}}-\frac{E \cdot C_{e q} \cdot k_{p}}{R \cdot C \cdot k_{4}}, \\
& F_{L}=\frac{E \cdot C_{e q} \cdot k_{d}}{C \cdot k_{4}} \cdot K \cdot K_{L}+\frac{E \cdot C_{e q} \cdot k_{p}}{C \cdot k_{4}}-\frac{E \cdot C_{e q} \cdot k_{d}}{R \cdot C^{2} \cdot k_{4}}-k_{E}, \\
& F_{m}=\frac{E \cdot C_{e q} \cdot k_{d}}{C \cdot k_{4}} \cdot K \cdot K_{E}-E \\
& F_{c 2}=\frac{E \cdot C_{e q} \cdot k_{d}}{C \cdot k_{4}} \cdot K \cdot 2 \cdot n \cdot I_{m}^{*}-2 \cdot n \cdot I_{L}^{*} .
\end{aligned}
$$

Use equation (10), then make the perturbation, Substitute (12) and (13) into the new state variable equation, meanwhile simplify it, then the following equation can be obtained:

$$
\frac{d \widehat{v_{c 2}}}{d t}=M_{o} \cdot \widehat{v_{o}}+M_{L} \cdot \widehat{i_{L}}+M_{m} \cdot \widehat{i_{m}}+M_{c 2} \cdot \widehat{v_{c 2}}
$$

Wherein,

$$
\begin{aligned}
& M_{o}=\frac{k_{i}}{k_{4}}+\frac{k_{d}}{k_{4}} \cdot \frac{1}{C} \cdot K \cdot K_{o}-\frac{k_{p}}{k_{4}} \cdot \frac{1}{R \cdot C}+\frac{k_{d}}{k_{4}} \cdot \frac{1}{R^{2} \cdot C^{2}} \\
& M_{L}=\frac{k_{p}}{k_{4}} \cdot \frac{1}{C}+\frac{k_{d}}{k_{4}} \cdot \frac{1}{C} \cdot K \cdot K_{L}-\frac{k_{d}}{k_{4}} \cdot \frac{1}{R \cdot C^{2}} \\
& M_{m}=\frac{k_{d}}{k_{4}} \cdot \frac{1}{C} \cdot K \cdot K_{E} \\
& M_{c 2}=\frac{k_{d}}{k_{4}} \cdot \frac{1}{C} \cdot K \cdot 2 \cdot n \cdot I_{m}^{*}
\end{aligned}
$$

From (12), (13),(14) and (15), the small signal full state equations are derived. It is supposed that the state function is $\dot{X}=A \cdot X$. So

$A=\left[\begin{array}{llll}K \cdot K_{o} & K \cdot K_{L} & K \cdot k_{E} & 2 \cdot K \cdot n \cdot I_{m}^{*} \\ \frac{F_{o}}{2 \cdot n \cdot L_{m} \cdot I_{L}^{*}} & \frac{F_{L}}{2 \cdot n \cdot L_{m} \cdot I_{L}^{*}} & \frac{F_{m}}{2 \cdot n \cdot L_{m} \cdot I_{L}^{*}} & \frac{F_{c 2}}{2 \cdot n \cdot L_{m} \cdot I_{L}^{*}} \\ \frac{1}{R \cdot C} & \frac{1}{C} & 0 & 0 \\ M_{o} & M_{L} & M_{m} & M_{c 2}\end{array}\right]$

\section{2)Controller Design}

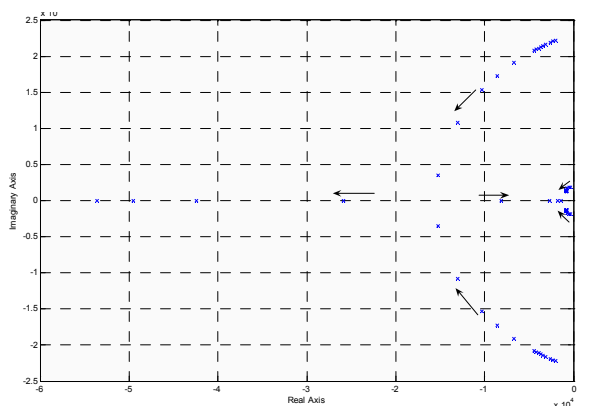

Fig. 3 Family of root locus for the following parameter: $k_{p}=0.8, k_{i}=1200, k_{d}=500 \mathrm{e}-6,1 \mathrm{e}-4<K_{4}<4 \mathrm{e}-2$

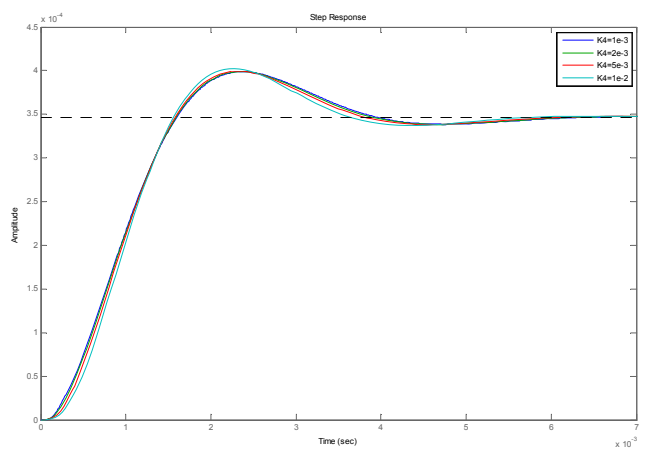

(a) $1 \mathrm{~V} /$ div and $200 \mathrm{mv} / \mathrm{div}$, Time: $200 \mathrm{~ms} / \mathrm{div}$

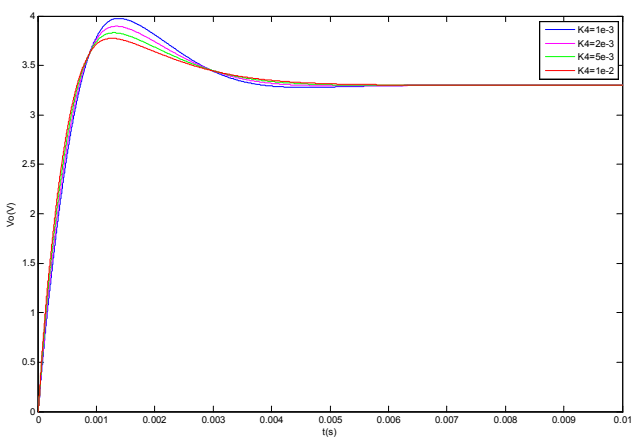

(b) 1 V/div

Fig.4 Comparison of large signal response and small signal response

In the control design, in order to achieve good transient response and stability of the system, the coefficients $k_{p}$, $k_{i}, k_{d}$ and $K_{4}$ are chosen from the pole placement. By this method, the effect of the control parameters over pole placement is illustrated. In Fig.3, the fours roots corresponding to the small signal model are placed. By choosing the appropriate parameters, the response of the system can be improved. Here we give the pole placement of $K_{4}$ as a example. By using the same method, the design of other parameters can be evaluated. Next, the averaged response generated by the large signal model (1)-(4) and the small signal model response generated by the model (12)-(15) are compared with each other by using the same controller parameters of $k_{p}, k_{i}, k_{d}$ and $K_{4}$. As shown in Fig. 4 (a), the converter output response is illustrated, in Fig. 4 (b), the response of the small signal model is illustrated. In both figures, the controller parameters are the same. It can be illustrated that, the responses are well matched. 


\section{EXPERIMENTAL RESULTS}

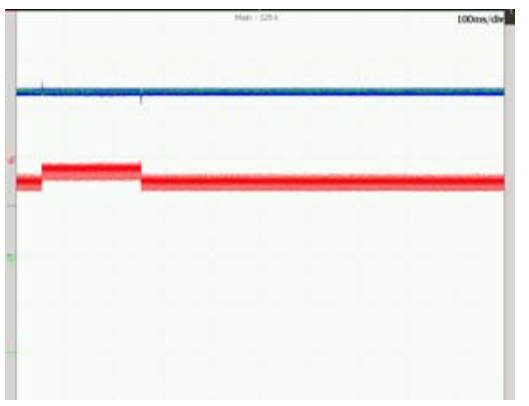

(a) $1 \mathrm{~V} /$ div and $200 \mathrm{mv} /$ div, Time: $200 \mathrm{~ms} / \mathrm{div}$

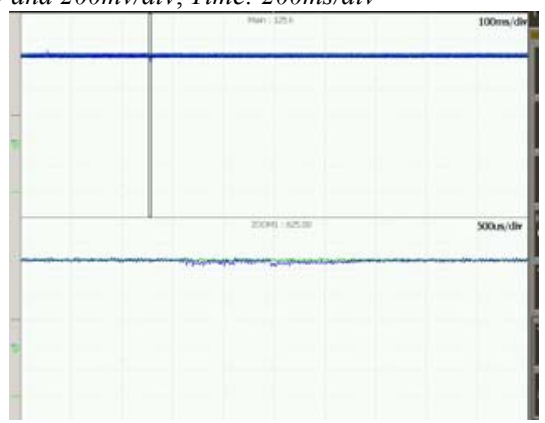

(b) 1 V/div

Fig.5 Experimental waveforms.

The controller is implemented by simple analog controller board. The rating output of the converter is as following: output voltage $3.3 \mathrm{~V}$, output current 10A. Fig.5 (a) shows the transient response of the output voltage and the output current, the dynamic change of the load is from $10 \mathrm{~A}$ to $5 \mathrm{~A}$, then to $10 \mathrm{~A}$. According to the waveforms, there is no current spike during the transient. In Fig.5 (b), the voltage response for the converter is enlarged. It can be seen that the transient response of the output voltage is very small and fast. The transient voltage of the converter is less than $0.1 \mathrm{~V}$. So, good dynamic response can be simply achieved by the SM controller. The proposed SM controller is robust and flexible for the converter.

\section{CONClusions ANd Future WORK}

This paper presents a novel sliding mode controller for the Half-bridge converter. The controller is robust and flexible for the converter. First, the small signal model and the controller design were presented. Then, the validity of the controller was verified by simulating and experimental results.

The controller is simply implemented in the analog controller board. By applying the novel sliding mode controller, the Half-bridge converter can achieve good transient response.

\section{REFERENCES}

[1] R. Venkataramanan, A. Sabanoivc, and S. C' uk, "Sliding mode control of DC-to-DC converters," in Proceedings,
IEEE Conference on Industrial Electronics, Control and Instrumentations (IECON), pp. 251-258, 1985.

[2] V. Utkin, J. Guldner, and J.X. Shi , Sliding Mode Control in Electromechanical Systems. London, U.K.: Taylor and Francis, 1999.

[3] S.C. Tan, Y.M. Lai, C.K. Tse, and M.K.H. Cheung, "A fixed-frequency pulse-width-modulation based quasisliding mode controller for buck converters", IEEE Transactions on Power Electronics, vol. 20, no. 6, pp. 1379-1392, Nov. 2005.

[4] S.C. Tan, Y.M. Lai, and C.K. Tse, "Implementation of pulse-width modulation based sliding mode controller for boost converters", IEEE Power Electronics Letter, vol. 3, no. 4, pp. 130-135, Dec. 2005.

[5] H. Li, D. Liu; F.Z. Peng, G.J. Su, "Small Signal Analysis of A Dual Half Bridge Isolated ZVS Bi-directional DC-DC converter for Electrical Vehicle Applications," in IEEE 2005 Power Electronics Specialists Conference, 2005, pp.2777-2782.

[6] H. Li, D. Liu, "Power Distribution Strategy of Fuel Cell Vehicle System with Hybrid Energy Storage Elements Using Triple Half Bridge (THB) Bidirectional DC-DC converter", in IEEE 2007 Industry Applications Conference,2007, pp.636-642

[7] V. Valchev, D. Yudov, J. Batchvarov, A. Van den Bossche, J. Melkebeek, "Zero-voltage and zero-current-switching half bridge DC/DC converter," in IEEE Industry Applications Conference, 2002, pp.2327-2334.

[8] L. Garcia de Vicuna, J.M. Guerrero, J. Matas, M. Castilla, J. Miret, "Simple nonlinear controller to reduce line and load disturbances in HBCC converter", Electronics Letters Volume: 38 , Issue: 25,2002, pp.1751-1752. 